\title{
Details of attention and learning change in first-episode schizophrenia
}

Research article

Christine Mohn ${ }^{a^{*}}$ and Anne-Kari Torgalsbøen ${ }^{b}$

a Research Department, Vestre Viken Hospital Trust, Drammen, Norway

b Department of Psychology, University of Oslo, Oslo, Norway

*Corresponding author: Christine Mohn, Research Department, Vestre Viken Hospital Trust,

Wergelands gate 10, 3005 Drammen, Norway.

Phone: + 47228451 15, Fax: + 27228450 01, e-mail: christinemohn@ hotmail.com

Word count: 3471 (excl. abstract, references, tables, and figures)

Word count abstract: 199

Number of tables: 4

Number of figures: 2

Running head: Detailed neurocognitive change in schizophrenia 


\begin{abstract}
Impaired attention and learning functions are common in schizophrenia. The details of this impairment, and how these change across time, are not well known. We aimed to compare the parameters of well-known attention and learning neuropsychological tests in first-episode schizophrenia (FES) patients and healthy controls in a 2-year follow-up period. The performance of 28-25 FES patients and pairwise matched healthy controls on the Continuous Performance Test-Identical Pairs, the revised Hopkins Verbal Learning Test, and the revised Brief Visuospatial Memory Test was compared at baseline and 2 years later. The attention dysfunction of the FES group was driven by slow reaction time and a comparative failure to identify correct hits. The reaction time was reduced somewhat across time in the patient group. Regarding the learning tasks, both groups increased their number of correct answers across trials. However, at each trial, the patient group exhibited lower scores, with a trend towards better visual learning performance across time. In summary, the FES patients were impaired in most of the parameters of the attention and learning tasks. Across time, modest improvements in reaction time and visual learning were displayed in the FES group. However, this group never caught up with the control group.
\end{abstract}

Key words: Attention, Neurocognitive function, Schizophrenia, Verbal learning, Visual learning 


\section{Introduction}

Neurocognitive dysfunction is a hallmark of schizophrenia spectrum disorders (Green and Harvey, 2014). Currently, one of the most widely used neurocognitive assessment batteries is the MATRICS Consensus Cognitive Battery (MCCB; Nuechterlein and Green, 2006). This battery consists of ten subtests, measuring seven cognitive domains. Each subtest is wellknown, has good psychometric properties, and has been used in a large variety of neuropsychological assessment studies for years (Nuechterlein et al., 2008). There is still, however, a need for studies of the details underlying the sum scores of the tests, e.g., whether a low sum score of a test of attention is the result of many false hits or slow reaction time.

Neurocognitive function may change due to illness progression or remission. Young, healthy controls may also exhibit cognitive changes due to naturally occurring brain development in adolescence and young adulthood, but these changes may not necessarily parallel those of schizophrenia patients. Recently, we have reported results from a 2-year follow-up study of first-episode schizophrenia (FES) patients and healthy controls matched on gender, age, and education level (Torgalsbøen et al., 2015). Both at baseline and at follow-up assessment, the FES group was significantly impaired compared to the control group on most cognitive tasks of the MCCB. In the current paper, we analyse the details of the attention/vigilance task (The Continuous Performance Test-Identical Pairs [CPT-IP], Cornblatt et al., 1988), the verbal learning task (Hopkins Verbal Learning Test-Revised [HVLT-R], Brandt and Benedict, 2001), and the visual learning task (Brief Visuospatial Memory Test-Revised [BVMT-R], Benedict, 1997). There are three reasons for this. First, in our previous study (Torgalsbøen et al., 2015), there were very large effect sizes separating the FES group and the control group on these tests. Second, these tasks are complex and consist of several parameters that each contribute to the sum score, and different subtasks may tap different cognitive processes. 
Third, the risk of practice effects are minimal. The MCCB was assembled from tests with high test-retest reliability, addressing the need for repeated testing in the same individuals. Among the tests of the MCCB, the CPT-IP has very high test-retest reliability ( $\mathrm{r} 0.84$, ICC 0.84 in a four-week period) (Nuechterlein and Green, 2006). For tests with lower test-retest reliability, such as the HVLT-R and the BVMT-R, alternate forms are used in the event of repeated testing. Thus, regarding these three instruments, the results at follow-up are much less likely to be biased by repeated exposure relative to what may be the case with other neuropsychological tests.

In summary, we wish to identify the details underlying the reduced capacity for attention and learning in FES patients compared to matched controls, and how these parameters change across a 2-year period. Several others have described cognitive changes across time in FES patients and healthy controls (Addington et al., 2005; Albus et al., 2006; Zhou et al., 2017). However, these studies report only the sum scores of the neuropsychological tasks. Our aim is to uncover the processes behind the sum scores. To the best of our knowledge, ours is the first study of this kind.

\section{Materials and methods}

\subsection{Participants}

Both the patient and the control samples (Table 1) are the ones that have been described in Torgalsbøen et al. (2014; 2015). Briefly, 28 first-episode schizophrenia patients were recruited consecutively from mental health facilities in the Oslo area. Inclusion criteria were age above 18 years, first-episode of illness within the schizophrenia and psychosis spectrum (DSM-IV; APA, 1994), and referral within the first 5 months of their first contact with mental health care. The baseline assessments for this study were carried out when the patients were in 
a stabilized illness period. Thus, the patients were undergoing their first episode of schizophrenia, but did no longer suffer from acute psychosis, and were capable of performing all the cognitive tasks. Exclusion criteria were affective disorders, head trauma, IQ below 70, and inability to understand spoken and written Norwegian. At the time of baseline testing, 16 patients $(57.0 \%)$ were hospitalised, and 12 were treated in out-patient clinics (43.0\%). Mean duration of untreated illness was 15.9 (SD 15.5) months. The diagnoses were as follows: 21 (75.0\%) had schizophrenia, $6(21.4 \%)$ had schizoaffective disorder, and 1 (3.6\%) had psychotic disorder NOS. Medicine dosages in the patient group were converted to a measure of the defined daily dose (DDD; the average maintenance dose per day for a drug used for its main indication in adults) (WHO, 2010).

The 28 healthy participants were recruited through senior high schools and newspaper and electronic advertisements in and around Oslo. The patients and controls were matched pairwise on gender, age, and education level (+/- one year). Written informed consent was obtained from all participants, and the study was approved by the Regional Committee for Medical Research Ethics (REK Sør-Øst).

At the 2-year follow-up assessment, 25 patients remained (Table 2). Of these, 3 (12.0\%) were hospitalised, $17(68.0 \%)$ in out-patient treatment, and $5(20.0 \%)$ treated by their general practitioner/first line health services. Correspondingly, the 3 healthy controls matching the 3 patients who dropped out were removed from the statistical analyses at follow-up.

(Insert Tables 1 and 2 here) 


\subsection{Clinical instruments}

The clinical interviews of the schizophrenia participants were carried out by an experienced clinical psychologist. Diagnoses were established using the Structural Clinical Instrument of Diagnosis for DSM-IV Axis I disorders (SCID-I), modules A-D. Symptom severity was assessed with the Positive and Negative Syndrome Scale (PANSS; Kay et al., 1987).

\subsection{Neuropsychological assessment}

This assessment was done by a clinical psychologist or graduate students of clinical psychology trained in neuropsychological testing. This study is part of a larger project, the Oslo Schizophrenia Recovery Study, where the Norwegian version of the MCCB (Nuechterlein and Green, 2006; 2009) is used to describe neurocognitive development in schizophrenia over a 10 year period. This battery consists of ten tests, and a complete test session usually lasts approximately 45-60 min. Norwegian reference data have been published (Mohn et al., 2012). From the MCCB, the three following tests are used in this study:

The CPT-IP (Cornblatt et al., 1988) requires the participant to monitor 2-, 3-, and 4-digit numbers on a computer screen and press a button when two identical digits are presented in a row. The 2-, 3-, and 4-digit numbers are presented in separate trials, and the participant may take breaks between these trials. Each trial score is the mean value of the signal-to-noise discrimination of that trial, and the sum score is the mean value of the signal-to-noise discrimination across all trials. A total sum score is calculated as the mean of all three trials. The task of CPT-IP lasts approximately $10 \mathrm{~min}$.

Verbal learning is assessed by the HVLT-R (Brandt and Benedict, 2001), which consists of a list of 12 words being read aloud three times at a pace of approximately $1 \mathrm{~s}$ per word. There 
are four words each representing three different groups of nouns: precious stones, animals, and housing. These words are presented randomly. At each time, the participant is asked to report back aloud the words that he/she remembers. The maximum score of each trial is 12 , and the maximum total score is 36 .

Visual learning is assessed by the BVMT-R (Benedict, 1997), in which the participant is asked to draw six geometrical figures after a $10 \mathrm{~s}$ exposure. This task also has three trials with a maximum score of 12 each, and a total sum score of 36 .

The raw scores of the cognitive tasks are reported.

In order to obtain reliable scoring of clinical and cognitive tests, the ratings of the different test administrators were compared. There was agreement in $94 \%$ of the cases. Cohen's kappa for this comparison was 0.69 , indicating good interrater reliability.

\subsection{Statistics}

All statistical analyses were performed with PASW Statistics 21. Group differences were analysed with MANOVAs and repeated measures ANOVAs. Effect size differences are reported as partial eta squared $\left(\eta^{2}\right)$.

\section{Results}

As previously reported (Torgalsbøen et al., 2015), all PANSS scores were significantly reduced from baseline to follow-up $(F \mathrm{~s}>39.00, p \mathrm{~s}<0.001)$. 
There were no statistically significant correlations between duration of untreated illness and any of the neuropsychological test variables.

The neurocognitive tests scores and the results of the ANOVAs are displayed in Tables 3 and 4. The last columns of these tables give the group significant tests and the group * time interaction effects. The significance tests of time are displayed in the rows below each pair of test scores. Moreover, the statistically significant group * time interaction effects of the cognitive tasks are presented in Figures 1 and 2.

\subsection{Group differences in CPT performance}

Both at baseline and follow-up, the schizophrenia group performed statistically significantly worse than the control group on almost all CPT variables, and the effect sizes were large. The largest group differences were found regarding the sum scores, number of correct hits, and the reaction time of the correct hits (Table 3).

For both groups, CPT performance was improved from baseline to 2-year follow-up with respect to the total sum score, number of false 2-digit responses, reaction time at false 2-digit responses, sum score at the 4-digit trial, and number of correct 4-digit hits (Table 3).

There were three statistically significant group * time interactions, in that the FES group exhibited faster reaction time at 2-, 3-, and 4-digit correct hits, whereas the control group became slower with time (Table 3 ).

(Insert Table 3 here) 


\subsection{Group differences in HVLT-R and BVMT-R performance}

Repeated measures analyses across the learning trials within each separate time point showed that statistically significant increases in scores occurred for both groups (HVLT-R: $F=$ 125.72, $p<0.001, \eta^{2} 0.70$; BVMT-R $\left.F=139.32, p<0.001, \eta^{2} 0.72\right)$. Hence, the repeated exposure had the desired learning effect for both groups.

Both at baseline and follow-up, the schizophrenia group performed statistically significantly worse than the control group on almost all HVLT-R and BVMT-R variables, again with large effect sizes. The largest group differences were seen on BVMT-R (Table 4).

For both groups, the HVLT-R sum score significantly deteriorated from baseline to 2-year follow-up (Table 4).

There were two statistically significant group * time interactions: The schizophrenia patients worsened their performance on HVLT-trial 1 from baseline to follow-up, while the control group remained stable. At BVMT-trial 2, the schizophrenia group improved from baseline to follow-up, while the control group was stable (Table 4).

(Insert Table 4 here)

At 2-year follow-up, 4 of the patients were defined as fully recovered (Torgalsbøen et al., 2015) (Table 2). It may be argued that the possibly improved cognitive performance of these participants would confound the results of the analyses. Therefore, the analyses were run again without the fully recovered participants. This procedure did not change the pattern of results displayed in Tables 3 and 4. 


\section{Discussion}

In this study of the details of the attention and learning tests of the MCCB, we uncovered several significant differences between FES patients and healthy controls. Although some improvement across time was observed, the FES patients mostly displayed the same deficiencies in the details of the cognitive tests at follow-up as at baseline. Hence, the FES patients start out at a lower level of performance, and the improvement they may display is not large enough to catch up with the controls.

It may be argued that practice effects due to repeated testing may account for the improvement seen in some of these cognitive parameters. However, the MCCB tests were assembled with the aim of avoiding this problem (Nuechterlein et al., 2008), and alternate versions of the stimulus material were used when available.

\subsection{The attention/vigilance task}

In the attention measure, i.e., the CPT-IP test, most of the group differences were statistically significant, with large effect sizes. The control group outperformed the FES group concerning all sum scores, correct hits, and the reaction times for correct hits. However, the number of false hits did not differ between the groups, although the patient group had significantly larger reaction times for false hits for 2- and 3-digit numbers. This implies that the reduced attentional function of the FES group was due to a comparative inability to identify correct targets as well as spending longer time at identification. The reaction time measures do not contribute directly to the CPT-IP sum scores, but indirectly they are relevant, as a rapid response is necessary for a correct hit to be made. If one is too slow, the opportunity of response is lost before the next target digit is presented. 
The fact that there were no significant group differences in the number of false hits, but in the false hits reaction times measures, suggest that the reduced attentional capacity of the FES group may not be related to faulty problem solving strategies as such at this early stage of information encoding. Our FES participants clearly understood the task at hand, but were too slow to respond in time to obtain high scores on the test in total.

For both groups, the pattern of response at the 2-year follow-up assessment was largely the same as at baseline. Over time, however, the FES group reduced their reaction time, resulting in significant group * time interaction effects. Hence, although both groups performed better on the CPT-IP task 2 years later compared to baseline, the FES group became faster at doing so.

\subsection{The learning tasks}

The results of the learning tests similarly present largely the same picture across groups at follow-up as at baseline. At both assessment points, both groups performed better with repeated administration of the stimulus material. However, in both the verbal (HVLT-R) and visual (BVMT-R) learning tests, the control group outperformed the FES group in most of the learning trials. This pattern was most consistent, and with larger effect sizes, in the BVMT-R test. This is an interesting finding, in that most previous studies have emphasized verbal learning dysfunction in schizophrenia (Mesholam-Gately et al., 2009).

Our learning tasks are more easily comparable than those of many other studies, in that they both demand immediate recall after three trials. However, the tasks are still too dissimilar for us to conclude that a genuine difference in visual and verbal learning occurs in schizophrenia. The HVLT-R requires only immediate verbal recall and may thus be relatively easy to 
perform, whereas the BVMT-R depends on the ability to draw as well as to remember the stimulus material. Hence, the larger group differences in visual learning could be due to this task being more difficult. A study of verbal and visual memory in schizophrenia using relatively comparable tasks reported similar findings to ours, in that the visual recall deficiencies were more pronounced than the verbal ones (Tracy et al., 2001). These results were also explainable in terms of the visual memory task being more difficult. Visual compared to verbal disadvantages have also been reported for adolescent psychosis patients (Øie and Rund, 1999; Øie et al., 1999). Thus, we suggest that the differences in verbal and visual learning/memory in our study may be attributed to task characteristics and not necessarily reflect genuine group differences in cognitive function.

Across time, there were certain small group differences in learning, as indicated by significant interaction effects in the first HVLT-R trial to the advantage of the control group, and in the second BVMT-R trial favouring the FES group. However, these changes were small, and taken together with the rest of the learning analyses do not suggest more than a trend towards altered patterns of the two learning tasks across time in the two groups.

In two studies not dissimilar to ours, Rodriguez-Sanchez et al. demonstrated that the overall cognitive function of their FES group was significantly lower compared to that of healthy controls, and that both groups in general displayed improved performance 1 (RodriguezSanchez et al., 2008) and 3 (Rodriguez-Sanchez et al., 2013) years after the baseline assessment. The exceptions were the verbal and visual learning test performances, which deteriorated across time in the FES group. The relevant tests used by Rodriguez-Sanchez et al. (2008; 2013) were the Rey Auditory Verbal Learning test (Schmidt, 1996) and the Rey 
Complex Figure test (Meyers and Meyers, 1995). Both of these are more difficult to perform than the HVLT-R and the BVMT-R, and this may account for the differences in results.

\subsection{Clinical implications}

Our results are particularly interesting due to the rapidly increasing use of the MCCB in studies of cognitive remediation in psychosis disorders (Green et al., 2014; Lystad et al., 2017). Cognitive remediation programs are usually strictly standardized, with the participants receiving the same therapeutic interventions at the same intervals. In the present study, however, we have demonstrated detailed cognitive changes in a group of FES patients undergoing a non-standardized treatment program, giving suggestions as to which changes may be expected from treatment tailored to each individual's clinical characteristics and relying on a combination of medication and psychotherapy.

Taken together, these findings suggest certain areas of cognitive intervention or remediation: The "natural" tendency of FES patients to display shorter reaction time at follow-up would make this parameter a useful target for exercises aimed at increasing cognitive function. Moreover, with respect to verbal learning and short term memory, efforts should be directed at encoding more stimuli early in the learning process. Finally, more improvement could be expected in the visual learning function compared to the verbal equivalent. On the other hand, cognitive remediation is a new type of intervention, and little is known of how much cognitive improvement it may generate in a long-term perspective. Following this line of reasoning, our study suggests not how large improvements are possible, but which processes are intact in FES patients across time. We have found that all detailed processes measured by the current attention and learning tasks are preserved, but that they are not fully functioning. 
The group difference is one of quantity, not quality. Detailed analyses of other domains would demonstrate if this is the case across all cognitive functions.

Recently, a study of the effects of cognitive remediation therapy on cognitive outcomes, assessed by the Norwegian MCCB, in schizophrenia spectrum disorders was conducted (Lystad et al., 2017). Verbal learning was the domain most improved after the intervention, replicating findings from international studies (McGurk et al., 2009; Christensen et al., 2014). Given that verbal learning and memory is assumed to be pivotal for functional outcome and ability to profit from rehabilitation (Green, 1996), these results are important. If the verbal learning function does not improve with conventional anti-psychotic treatment, as our data suggest, cognitive remediation may be more efficient.

\subsection{Strengths and limitations}

A major strength of this study is the participation of FES patients whose cognitive performance is not affected by years of medication or other types of treatment. Another strong aspect is our gender, age, and education pairwise matched control group, avoiding the most relevant confounding demographic variables. Moreover, the participants completed all the tests at the assessment points, and the attrition rate is low.

The limitations all concern the relatively modest sample sizes. First, larger samples would have permitted more confidence in our statistical calculations, in particular with respect to the time differences. The group differences had, however, large effect sizes, which indicate few false positives. 
Second, we did not control for medication effects or the effect of general intellectual function (IQ). However, in a previous study of the same participants, these variables had no statistically significant relationship to the neurocognitive scores (Torgalsbøen et al., 2014). Relatedly, we did not control for the influence of illness severity on the test results. The relationship between positive psychosis symptoms and sum scores of cognitive function has normally been found to be small (Dominguez et al., 2009). However, significant associations between negative symptoms and cognitive test results have been reported (Anda et al., 2016; Dominguez et al., 2009). We can therefore not conclude that symptom levels have no relation to the detailed cognitive processes we studied here. These associations should be investigated in a larger sample.

Third, there may be a hierarchical relationship between attention and learning, in that a fully functioning attention capacity is necessary for learning to occur. Hence, some of the variance in the learning outcome parameters may have been explainable in terms of deficient attention processes. In such cases, cognitive remediation should be directed at improving attentional functions, and enhanced learning strategies will naturally follow. With larger samples, we would be able to analyse the influence of attention on learning, and draw firmer conclusions as to the clinical utility of our findings.

\subsection{Conclusion}

The schizophrenia patients were impaired in most of the parameters of the attention and learning tasks at both assessment points. Modest improvements in reaction time and visual learning were observed in the patient group, but this group never caught up with the control group in terms of neurocognitive function. 


\section{Conflict of interest}

None of the authors has any conflict of interest.

\section{Author contributions}

AKT designed the study, wrote the protocol, performed or supervised the clinical and neuropsychological assessments, and contributed to writing the manuscript. CM performed the statistical analyses and drafted the manuscript. Both authors had full access to all data and have read and approved the final manuscript.

\section{Funding}

This project was funded by the Helse Sør- $\emptyset$ st (Health South East) Regional Hospital Trust, project number 2009044, and the Department of Psychology, University of Oslo.

\section{Acknowledgements}

Dr Merete Glenne Øie is gratefully acknowledged for her valuable comments to an earlier version of this paper. 


\section{References}

Addington, J., Saeedi, H., Addington, D., 2005. The course of cognitive functioning in first episode psychosis: Changes over time and impact on outcome. Schiz. Res. 78 (1), 35-43.

Albus, M., Hubmann, F., Mohr, F., Hecht, S., Hinterberger-Weber, P., Sietz, N.-N., et al., 2006. Neurocognitive functioning in patients with first-episode schizophrenia. Eur. Arch. Psychiatry Clin. Neurosci. 256 (7), 442-451.

American Psychiatric Association, 1994. Diagnostic and Statistical Manual of Mental Disorders. APA, Washington, DC.

Anda, L., Brønnick, K.S., Johnsen, E., Kroken, R.A., Jørgensen, H., Løberg, E.-M., 2016. The course of neurocognitive changes in acute psychosis: Relation to symptomatic improvement. PLoS ONE 11 (12), e0167390.

Benedict, R.H.B., 1997. Brief Visuospatial Memory Test - Revised. Psychological Assessment Resources, Odessa, FLA.

Brandt, J., Benedict, R.H.B., 2001. The Hopkins Verbal Learning Test - Revised. Psychological Assessment Resources, Odessa, FLA.

Christensen T.O., Vesterager L, Krarup, G., Olsen, B.B., Melau, M., Gluud, C., et al., 2014. Cognitive remediation combined with an early intervention service in first episode psychosis. Acta Psychiatr. Scand. 130 (4), 300-310. 
Cornblatt, B.A., Risch, N.J., Faris, G., Friedman, D., Erlenmeyer-Kimling, L., 1988. The continuous performance test, identical pairs version (CPT-IP): I. New findings about sustained attention in normal families. Psychiatry Res. 26 (2), 223-238.

Dominguez, M. dG., Viechtbauer, W., Simons, C. J. P., van Os, J., Krabbendam, L., 2009. Are psychotic psychopathology and neurocognition ortogonal? A systematic review of their associations. Psychol. Bull. 135, 157-171.

Green, M.F., 1996. What are the functional consequences of neurocognitive deficits in schizophrenia? Am. J. Psychiatry 153 (3), 321-330.

Green, M.F., Harris, J.G., Nuechterlein, K.H., 2014. The MATRICS Consensus Cognitive Battery: What we know 6 years later. Am. J. Psychiatry 171 (11), 1151-1154.

Green, M.F., Harvey, P.D, 2014. Cognition in schizophrenia: Past, present, and future. Schiz. Res.: Cogn. 1 (1), e1-e9.

Holmén, A., Juuhl-Langseth, M., Thormodsen, R., Melle, I., Rund, B.R., 2010. Neuropsychological profile in early-onset schizophrenia-spectrum disorders: measured with the MATRICS Battery. Schiz. Bull. 36 (4), 852-859.

Kay, S.R., Fiszbein, A., Opfer, L.A., 1987. The Positive and Negative Syndrome Scale (PANSS) for Schizophrenia. Schiz. Bull. 13 (2), 261-276. 
Lystad, J.U., Falkum, E., Haaland, V.Ø., Bull, H., Evensen, S., McGurk, S.R., et al., 2017.

Cognitive remediation and occupational outcomes in schizophrenia spectrum disorders: A 2year follow-up study. Schiz. Res. 185 (1), 122-129.

McGurk, S.R., Mueser, K.T., DeRosa, T.J., Wolfe, R., 2009. Work, recovery, and comorbidity in schizophrenia: a randomized controlled trial of cognitive remediation. Schiz. Bull. 35 (2), 319-335.

Mesholam-Gately, R.I., Giuliano, A.J., Goff, K.P., Faraone, S.V., Seidman, L.J., 2009. Neurocognition in first-episode schizophrenia: A meta-analytic review. Neuropsychol. 23 (3), 315-336.

Meyers, J., Meyers, K., 1995. Rey Complex Figure and Recognition Trial: Professional manual. Psychological Assessment Resources, Odessa, FLA.

Mohn, C., Sundet, K., Rund, B.R., 2012. The Norwegian standardization of the MATRICS (Measurement and Treatment Research to Improve Cognition in Schizophrenia) Consensus Cognitive Battery. J. Clin. Exp. Neuropsychol. 34 (6), 667-677.

Nuechterlein, K.H., Green, M.F, 2006. MCCB. MATRICS Consensus Cognitive Battery. Manual. MATRICS Assessment Inc, Los Angeles, CA.

Nuechterlein, K.H., Green, M.F., 2009. MATRICS Consensus Cognitive Battery, Norwegian Version. (Rund BR, Sundet KS, trans.) MATRICS Assessment Inc, Los Angeles, CA. 
Nuechterlein, K.H., Green, M.F., Kern, R.S., Baade, L.E., Barch, D.M., Cohen, J.D., et al., 2008. The MATRICS consensus cognitive battery, part 1: Test selection, reliability, and validity. Am. J. Psychiatry 165 (2), 203-213.

Øie, M., Rund, B.R., 1999. Neuropsychological deficits in adolescent-onset schizophrenia compared with attention deficit hyperactivity disorder. Am. J. Psychiatry 156 (8), 1216-1222.

Øie, M., Sundet, K., Rund, B.R., 1999. Contrasts in memory functions between adolescents with schizophrenia or ADHD. Neuropsychologia 37 (12), 1351-1358.

Rodriguez-Sanchez, J.M., Ayesa-Arriola, R., Perez-Iglesias, R., Perianez J.A., MartinezGarcia, O., Gomez-Ruiz, E., et al., 2013. Course of cognitive deficits in first-episode of nonaffective psychosis: A 3-year follow-up study. Schiz. Res. 150 (1), 121-129.

Rodriguez-Sanchez, J.M., Perez-Iglesias, R., Gonzalez-Blanch, C., Pelayo-Teran, J.M., Mata, I., Martinez, O., et al., 2008. 1-year follow-up study of cognitive function in first-episode nonaffective psychosis. Schiz. Res. 104 (1-3), 165-174.

Schmidt, M., 1996. Rey Auditory Verbal Learning Test: A Handbook. Western Psychological Services, Los Angeles, CA.

Torgalsbøen, A.-K., Mohn, C., Czajkowski, N., Rund, B.R., 2015. Relationship between neurocognition and functional recovery in first-episode schizophrenia: Results from the second year of the Oslo multi-follow-up study. Psychiatry Res. 227 (2-3), 185-191. 
Torgalsbøen, A.-K., Mohn, C., Rund, B.R., 2014. Neurocognitive predictors of remission of symptoms and social and role functioning in the early course of first-episode schizophrenia. Psychiatry Res. 216 (1), 1-5.

Tracy, J.I., Mattson, R., King, C., Bundick, T., Celenza, M.A., Glosser, G., 2001. A comparison of memory for verbal and non-verbal material in schizophrenia. Schiz. Res. 50 (3), 199-211.

WHO. Collaborating Centre for Drug Statistics Methodology. DDD Definitions and General Considerations, 2010. http://www.whocc.no/ddd/definition_and_general_considera/ (Accessed 01.08.16.)

Zhou, F.-C., Wang, C.-Y., Ungvari, G.S., Ng, C.H., Zhou, Y., Zhang, L. et al., 2017. Longitudinal changes in prospective memory and their clinical correlates at 1-year follow-up in first-episode schizophrenia. PLoS ONE 12 (2), e0172114. 
Table 1. Demographic characteristics of the participants at baseline.

\begin{tabular}{|l|l|l|}
\hline & Patients $(\mathbf{n = 2 8})$ & Controls $(\mathbf{n = 2 8})$ \\
\hline Age in years & $21.0(2.6)$ & $21.1(2.7)$ \\
\hline Gender & $11(60.7 \%)$ men & $17(60.7 \%)$ men \\
Elementary school & $11(39.3 \%)$ & $11(39.3 \%)$ women \\
High school & $8(28.6 \%)$ & $9(32.1 \%)$ \\
Some college & $7(25.6 \%)$ & $1(3.6 \%)$ \\
BA degree or higher & $2(7.2 \%)$ & $1(3.6 \%)$ \\
\hline
\end{tabular}

Age in mean $(S D)$. 
Table 2. Clinical characteristics of the patients at baseline and 2-year follow-up.

\begin{tabular}{|l|l|l|}
\hline & Baseline (n=28) & 2 years (n=25) \\
\hline In remission & - & $16(64.0 \%)$ \\
\hline Fully recovered & - & $4(16.0 \%)$ \\
\hline Substance abuse at time of testing & $1(3.6 \%)$ & $3(12.0 \%)$ \\
\hline $\begin{array}{l}\text { Medication } \\
\text { First generation antipsychotics }\end{array}$ & $5(18.5 \%)$ CDD: 1.06 & $3(12.0 \%)$ CDD: 0.60 \\
Antidepressants & $22(79.7 \%)$ CDD: 0.83 & $16(64.0 \%)$ CDD: 1.00 \\
Mood stabilizers & $11(39.3 \%)$ CDD: 1.24 & $10(40.0 \%)$ CDD: 1.10 \\
Anticonvulsants & $-31.1(16.7)$ & $3(12.0 \%)$ CDD: 3.9 \\
No medication & $21.2(4.8)$ & $3(12.0 \%)$ CDD: 0.4 \\
Total & $40.2(9.3)$ & $4(16.0 \%)$ \\
\hline PANSS & $19.7(5.7)$ & $12.0(6.4)$ \\
\hline $\begin{array}{l}\text { Positive } \\
\text { Negative }\end{array}$ & $24.2(6.6)$ \\
\hline
\end{tabular}

PANSS scores in mean $(S D)$. CDD: Calculated daily dose of medication based on the prescribed dosage divided by the defined daily dosage. 
Table 3. Performance on the CPT-IP.

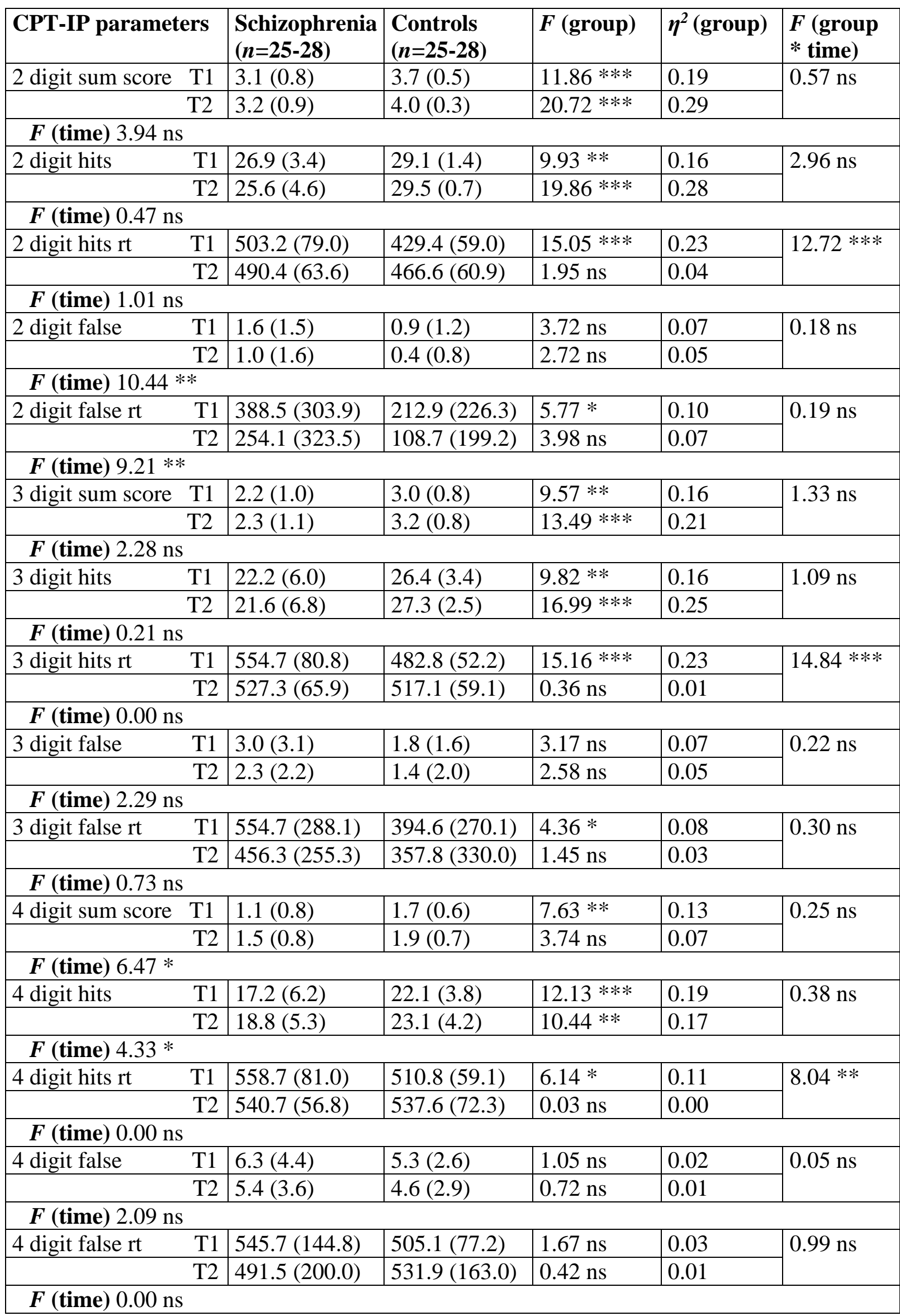




\begin{tabular}{|c|c|c|c|c|c|c|}
\hline Total sum score & T1 & $2.0(0.6)$ & $2.8(0.5)$ & $29.00 * * *$ & 0.36 & \multirow[t]{2}{*}{$0.64 \mathrm{~ns}$} \\
\hline & $\mathrm{T} 2$ & $2.4(0.8)$ & $3.0(0.5)$ & $12.99 * * *$ & 0.20 & \\
\hline
\end{tabular}

Hits and false: total numbers of correct and incorrect identification, respectively. rt: reaction time, in ms. T1: Baseline, T2: 2-year follow-up. $F$ (time): Within group significance test. $F$ (group): Between group significance test. $F$ (group * time): Significance test of interaction effect. $\eta^{2}$ : effect size. ${ }^{* * *}: p<0.001, * *: p<0.01, *: p<0.05$, ns: non-significant. 
Table 4. Performance on the HVLT-R and BVMT-R.

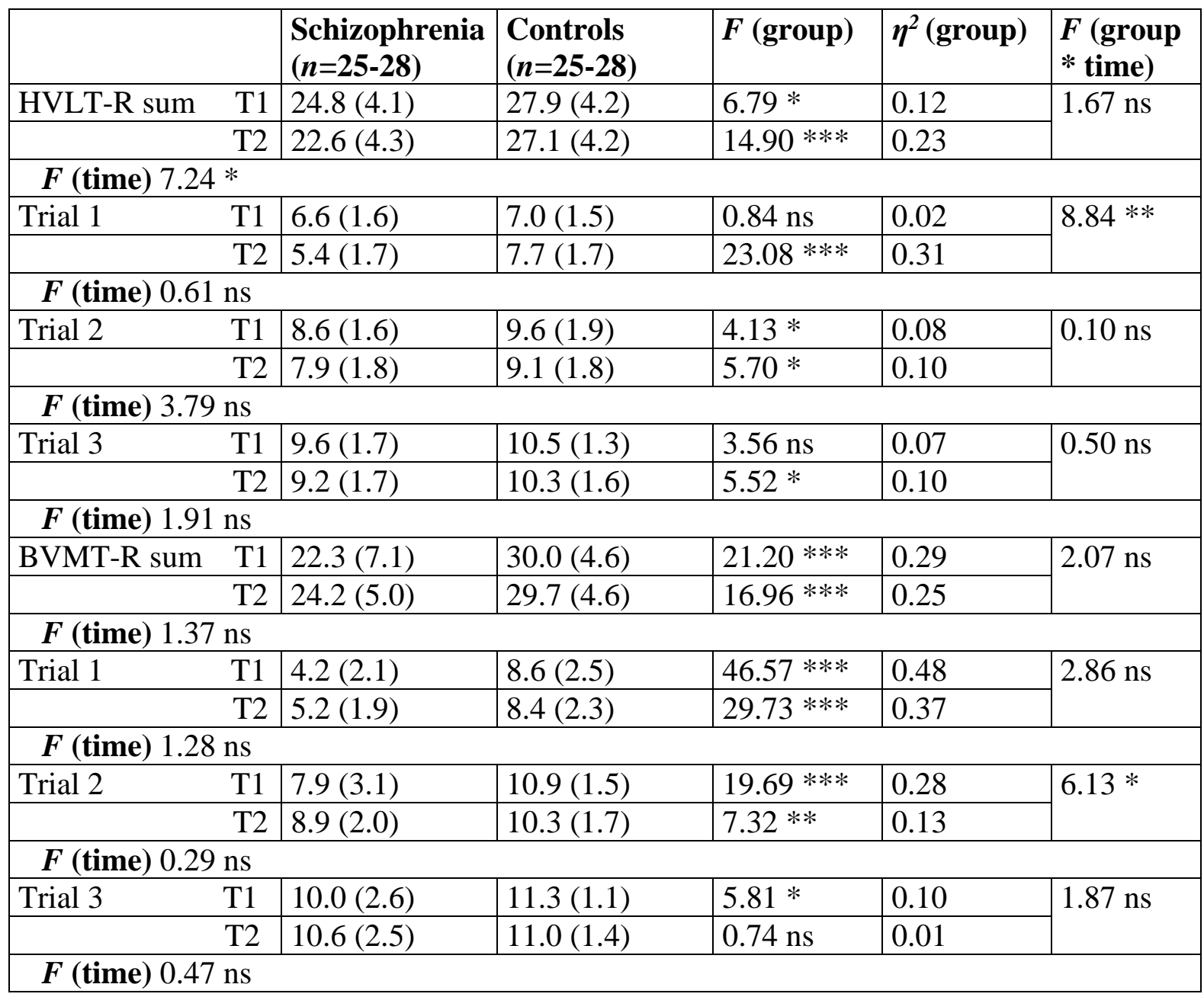

T1: Baseline, T2: 2-year follow-up. $F$ (time): Within group significance test. $F$ (group):

Between group significance test. $F$ (group $*$ time): Significance test of interaction effect. $\eta^{2}$ : effect size. ${ }^{* * *}: p<0.001,{ }^{* *}: p<0.01, *: p<0.05$, ns: non-significant. 
Figure 1.The results of the statistically significant interaction effects from the CPT-IP task.
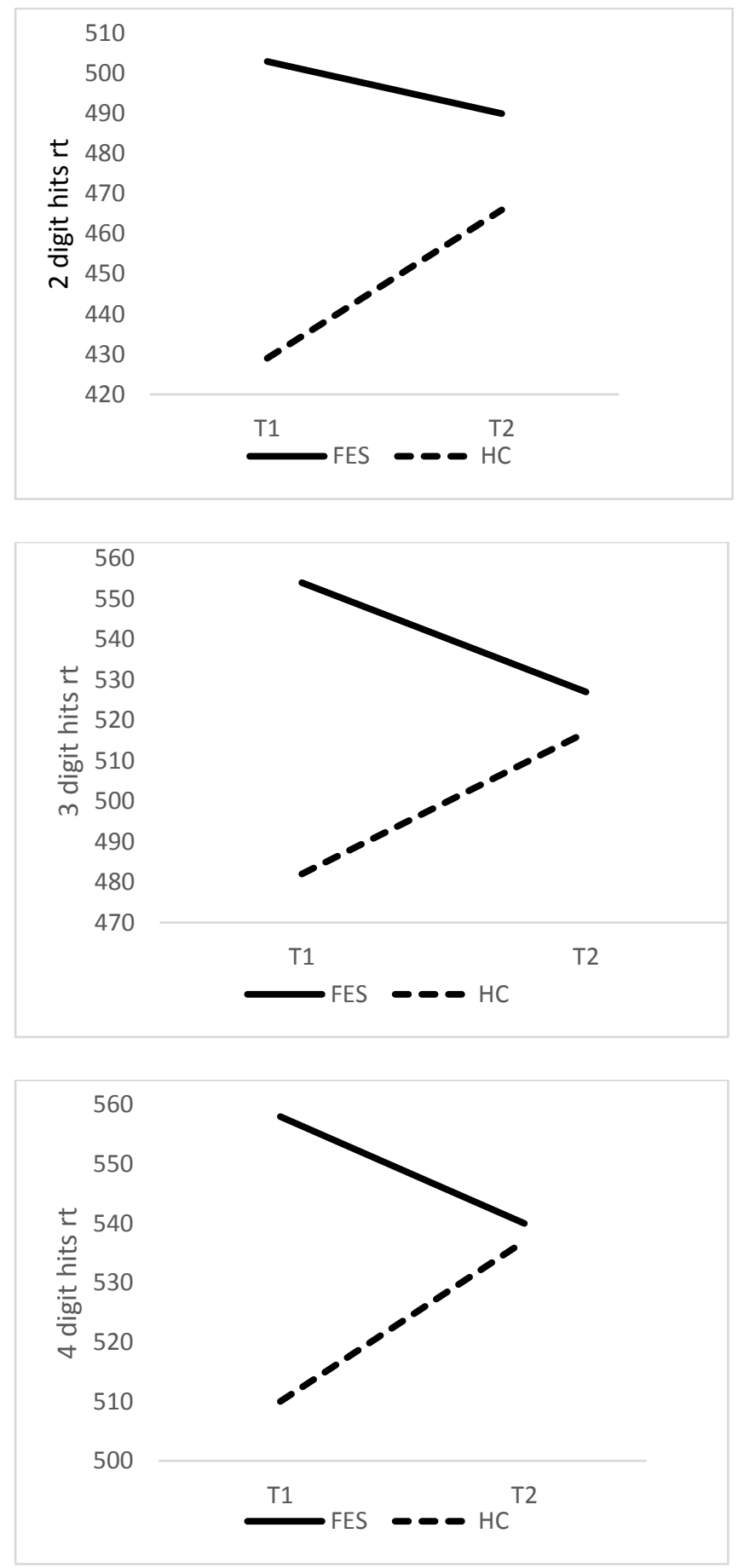

FES: First episode schizophrenia $(n=25-28)$. HC: Healthy controls $(n=25-28)$. T1: Baseline, T2: 2-year follow-up. The numbers of the $\mathrm{Y}$ axis denote mean reaction time in $\mathrm{ms}$. 
Figure 2. The results of the statistically significant interaction effects from the HVLT-R and BVMT-R tasks.
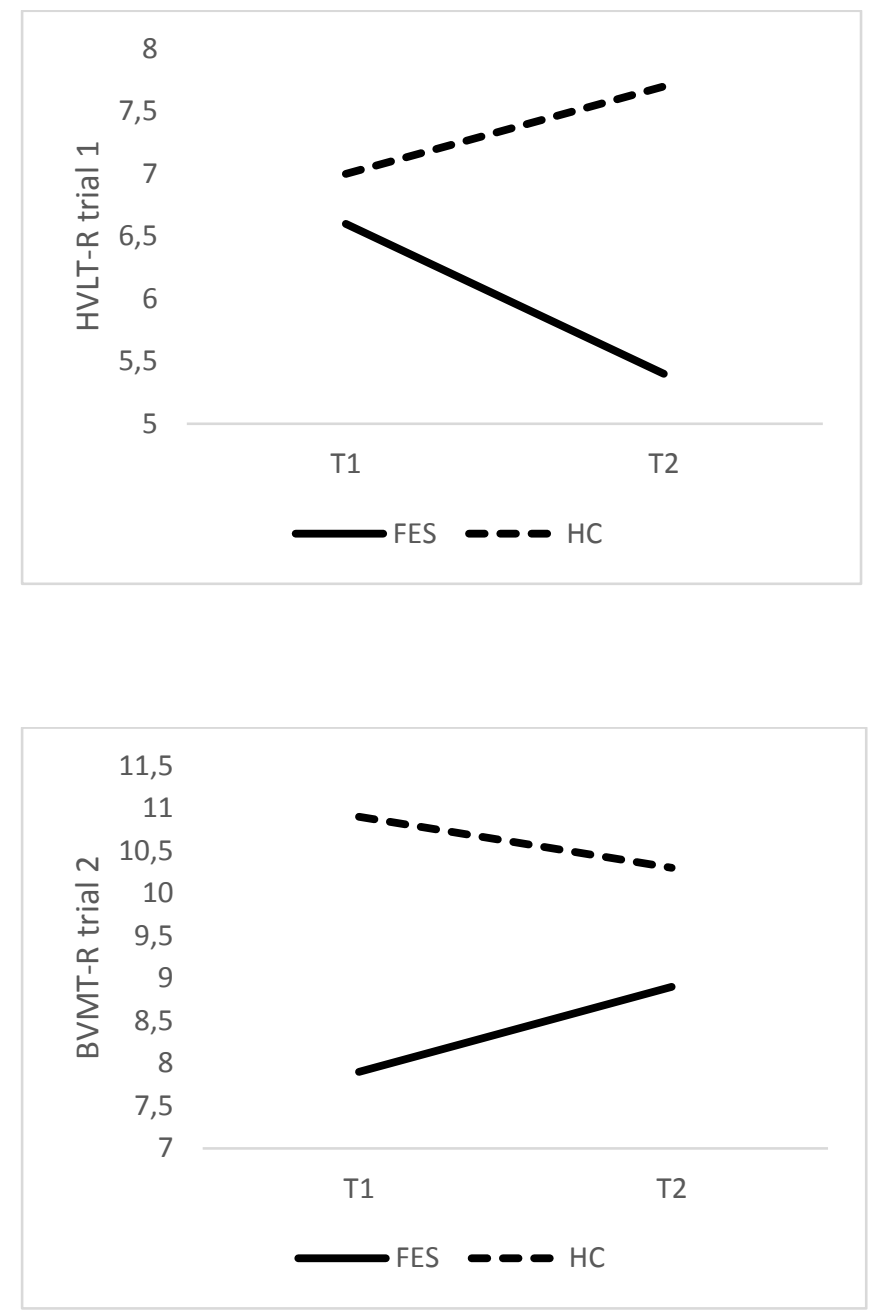

FES: First episode schizophrenia $(n=25-28)$. HC: Healthy controls $(n=25-28)$. T1: Baseline, T2: 2-year follow-up. The numbers of the $\mathrm{Y}$ axis denote mean number of words (HVLT-R) and figures (BVMT-R) remembered. 
